\title{
Coma barbitúrico terapéutico en el niño
}

\author{
Dr. León Ad̛lerstein S. ${ }^{1}$; Dr. Walter Kaufmann R. ${ }^{\text {; }}$ Dr. Juan C. Faúndez L. ${ }^{2}$ \\ Therapeutic barbiturate coma in children
}

\begin{abstract}
High dose barbiturate therapy has been used, with good results, in the 1reatment of intracraneal hypertension, anoxic ischemic encephalopathy and status epilepticus. Two groups of patients treated with thiopental were analized. The first group comprised 10 patients with status epilepticus, refractory to other therapy: all of them had a fast, safe and successful response. The second group included 9 patients, with either intracrarseal hypertension or hypoxic-ischemic encephalopathy of various ethiologies or both. The best results were obtained in near drowning cerebral anoxia, and cardiorespiratory arrest. Treatment protocols, cotateral effects, mortality and neurologic sequelae are discused. Therapeutic barbiturate coma in children, must be applicd in intensive care units, because mechanic ventilation, continuos monitoring of intracraneal pressure (in patients with intracraneal hypertension), electroencephalography and evoked potentials, are necessary for adequate evaluation and control of the patients.

(Key words: Barbiturate coma, hypoxic-ischemic encephajopathy, status epilepticus, intracraneal hypertension, ref Jactory, seizures, thiopental, treatment).
\end{abstract}

Desde hace algunos años, ha surgido un interés creciente en el uso de barbitúricos en altas dosis, en afecciones del sistema nervioso central, asociadas a encefalopatía hípóxico-isquémica, hipertensión endocraneana y estado epiléptico.

Las encefalopatías hipóxica-isquémicas incluyen paro cardiorespiratorio (accidentes anestésicos y secundarios a "shock" de diferentes etiologías), accidentes vasculares encefálicos y asfixia por inmersión. En las condiciones clínicas en que isquemia $y$ anoxia son los trastornos principales, la protección barbitúrica del cerebro humano no ha sido comprobada, sin embargo, las experiencjas en animales ${ }^{1-4}$ han demostrado su beneficio, especialmente si se cumplen en forma preventiva lo que ha impulsado al uso clínico del coma barbitúrico.

Experiencias clínicas en casos de hipertensión endocraneana han demostrado, mediante observación continua de la presión intracraneana, que los barbitúricos son capaces de reducirla, espe-

1. Departamento de Neurología infantil, Hospital Roberto del Río.

2. Servicio de Pediatría, Hospital Roberto del Rio. cialmente, en sindrome de Reye y en los traumatismos encéfalocraneanos severos ${ }^{6-12}$; probablemente porque reducen el flujo sanguíneo cerebral y el metabolismo basal del sistema nervioso central.

Estudios nacionales y extranjeros ${ }^{13-17}$, han demostrado la utilidad clínica de los barbitúricos en alta dosis, en el manejo del estado epiléptico.

No es infrecuente que los problemas recién mencionados se combinen, llevando al paciente a situaciones de tal gravedad que hacen plantear al coma barbitúrico como única alternativa tera. péutica. Sin embargo, su uso no debe ser indiscriminado y requiere un manejo muy complejo de] paciente, en unidades que cuentan con infraestructura adecuada.

En este trabajo se describe la experiencia acumulada en el uso de barbitúricos, en altas dosis (thiopental), en el hospital Roberto del Rio, con el propósito de aclarar algunas dudas que. con su empleo cada vez más frecuente, han aparecido sobre su aplicación y efectividad.

\section{MATERIAL Y METODO}

Se amalizaron 19 pacientes que ingresaron a la unidad 
de cuidados especiales, del servicio de pediatría, del hospital Roberto del Río, en el periodo comprendido entre enero de 1983 y junio de 1984 en quienes se indicó coma barbitúrico terapéutico. Diez pacientes (Grupo I, Tabla 1) sufrían estados convulsivos de diversas etiologias înfecciones meningoencefálicas, traumatismos encéfalocraneanos, epilepsia y trastornos metabólicos) y nueve (Grupo II, Tabla 2) eran pacientes con síndrome de hipertensión endocraneana, encefalopatía hipoxico-isquémica (asfixia por inmersión, paro cardiorespitatorio, síndrome de Reye $y$ encefalitis) o ambas.

En el Grupo [ se usó thiopental sódico 2 ó $4 \% \mathrm{cr}$ solución de glucosa al $5 \%$ comenzando con bolos de $I$ a $17 \mathrm{mg} x$ ke para suprimir la crisis convulsiva, continuando con dosis de mantención de 1 a $6 \mathrm{mg} \mathrm{x} \mathrm{kg} \mathrm{"} x h^{\prime}$, en microgoteo. La duración del tratamiento varió eлtre 2,5 y 128 horas, dependiendo de la respuesta al medicamento.

En el Grupo II la dosis inicial varió entre 2,5 y $9 \mathrm{mg}$ $x \mathrm{~kg}$, buscando obtener coma profundo, sin alterar parámetros vitales. La dosis de matjtención fue de 1 a $12 \mathrm{hg} x \mathrm{~kg} x$ hora. durando el tratumiento entre $40 \mathrm{~min}$ (por muerte del paciente) y 131 horas.

Los parámetros usados para regular la dosis del coma batbitúrico fueron: presión arterial, frecuencia catdíuca, temperatura, convulsiones, posturas anormales (sineinesias extenxores y thexoras), reflejos integuados en el tronco cerebral (respuesta fotomotora, respuesta óculo-cefálica, respuesta óculovestibular, reflejo corneal), reflejos osteotendinosos y ton muscular. En ningún paciente fuc posible vigilar la presión intracranoana. En todos los pacientes se emplea ventilación mecátrica mientras duró el coma batbitúrico. La suspensión del tratamiento en el Grupo I se realizó después de transcurrir 24 horas sin que ocutrieran convulsiones, procediendo a retirar progresivamente el thiopental. Fin el Grupo II, \$ usó el tratamiento por un período mínimo de 48 hrs. y luego se retiró en forma progresiva, rejnstalándolo si réaparecían signos indirectos de hipertensión endocrantiana.

En las Tablas 1 y 2 se especiticia el rangu de edades de los pacientes y el tratumiento previo al coma barbiturico.

\section{RESULTADOS}

E] Grupo I, con estado convulsivo (Tabla 1) incluye 10 pacientes, 7 eran varones, sus edades variaron entre 3 meses a 10 años. Las enferme. dades de base fueron: 2 meningitis bacterianas agudas; I meningoencefalitis; I shock septico, cuya ctiología no fue precisada; 2 epilepsias generalizadas; 1 intoxicación salicílica; 1 traumatismo encéfalocraneano agudo cerrado; 1 síndrome hemolítico urémico y 1 insuficiencia renal.

La duración de las convulsiones, antes de la administración de thiopental, varió entre $20 \mathrm{~min}$ y 5 horas. La desaparición de las crisis se obtuvo en forma casi innediata en todos nucstros pacientes. Luego. se prolongó el uso de thiopental en dosis mínimas, sin convulsiones, por 24 hrs., altes de retirar el medicamento progresivamente. En dos pacientes reaparecieron crisis convulsivas dentro de las primeras 24 hrs. después de la suspension del tratamiento.

La lctalitad fue de $\mathbf{4}$ pacientes y las secuclas neurológicas ocurrieron en un número similar, lo cual puede atribuírse a su enlermedad de base. Al egreso, de 4 niños con daño neurológico, uno tenía daño previo, el que persistió con iguales características a su alta y los otros 3 presentaban un sindrome piramidal crónico bilateral de diferente magnitud, con retardo del desarrollo psicomotor en todos, asociándose en uno de ellos síndrome t,e West y en otro hid rocefalia secunda. ria a mer ingitis.

De dos pacientes que presentaron estado convulsivo ascriado a epilepsia, en uno este fue la primera evidencia de la enfermedad. En el segun.

Tabla 1 .

Coma barbitúrico terapéulico (thiopental en estado eonvulsivo

\begin{tabular}{|c|c|c|c|c|c|c|c|c|c|c|}
\hline 1. $S \times x+3$ & $M$ & $M$ & $M$ & Ii & M & NI & ]. & M & $M$ & t \\
\hline 2. Jidind & $7 m$ & $3 m$ & $1 \mathrm{~d} 6 \mathrm{~mm}$ & $1: 9 m$ & $422 m$ & 235 & $\left.2 x^{\prime}\right)$ & $30 \mathrm{u}$ & 2 alula & 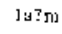 \\
\hline 3 Niaguorticu & $M B A$ & MHA & M. E & Sc゙ & ]:PI & $|\cdot P|$ & إنS .اמו & TLC. ('Ess & Sillı & I.R \\
\hline 4. $\mathbf{T}(p)$ (x) (x) & III: irda & If $;$; vit & (; & : & ; & is & i. & i & di & (i \\
\hline $\begin{array}{l}\text { 5. Duracion de :intry. } \\
\text { prevki a CRT Ihvias? }\end{array}$ & 1 & 2 & 4 & 0,5 & 0,3 & 0,8 & l.,5 & G & 19,8 & $s$ \\
\hline $\begin{array}{l}\text { funcleina } \\
\text { diatepara }\end{array}$ & + & $\begin{array}{l}t \\
t\end{array}$ & $\begin{array}{l}+ \\
+ \\
+\end{array}$ & $\stackrel{+}{+}$ & $\begin{array}{l}-1 . \\
+ \\
-\end{array}$ & $\stackrel{+}{+}$ & $\stackrel{+}{+}$ & $\stackrel{+}{+}$ & $\stackrel{+}{+}$ & $\stackrel{+}{+}$ \\
\hline $\begin{array}{l}\text { 7. Jhosis ntijntcheión } \\
\text { ptometojo isug } \times \mathrm{kg} \times \text { holal }\end{array}$ & 2 & 17 & 3,6 & 3.5 & 5 & 5 & 3 & 1 & 5 & 5 \\
\hline $\begin{array}{l}\text { R. Posis manterición } \\
\text { proniedio dnip } x \text { kx } x \text { hora }\end{array}$ & 2,9 & 62 & 3,7 & 1,5 & 1 & $5, ?$ & 15 & 1,2 & 3 & 3.4 \\
\hline 9. Durackon $C \mathrm{BT}$ (horis) & 60,5 & 96 & $8 y$ & 128 & 24 & 43,5 & 24 & 42 & 2.5 & 78 \\
\hline 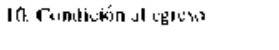 & b & I) & (3) & $\mathbf{I}$ & $N$ & 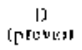 & $\mathrm{T}$ & $y$ & f: & [i \\
\hline 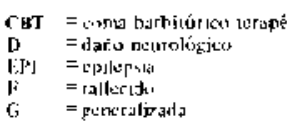 & & & $\begin{array}{l}\text { He: }= \\
\text { Mish }= \\
\text { N }- \\
\text { MI. - } \\
\text { S.S - }\end{array}$ & 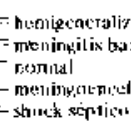 & a isudis & & $\begin{array}{l}\text { SEt } \\
\text { IR }\end{array}$ & $\begin{array}{l}=\text { in } 1,04 \\
=\text { iittdr } \\
=\text { irsul }\end{array}$ & 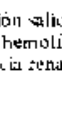 & rimico \\
\hline
\end{tabular}


do caso el paciente tenía una parálisis cerebral espastica y epilepsia secundaria a una encefalitis que sufíió a los 2 meses de edad.

En cuanto a las características de la crisis convulsiva en nuestros 10 pacientes, 8 presentaron crisis generalizadas, que a veces lacteralizaban $y$ en dos fueron crisis hemigeneralizadas izquierdas.

En el Grupo Il (Tabla 2) se incluyeron 9 pacientes, 5 eran mujeres. Las edades variaron entre 2 meses y 13 años. Los diagnósticos de base fueron: 3 asfixias por inmersión, 3 paros cardiorespiratorios ( 1 por accidente anestésico, 1 secun. dario a shock hipovolémico en cetoacidosis diabética y 1 secundario a neumopatia aspirativa). 2 sindromes de Reye $y$ l encefalitis posiblemente herpética (no confirmada).

En los 3 pacientes con asfixia por inmersión, el tratamiento con thiopental se inició al ingreso al hospital (entre 0,5 y $I$ horas despues de] accidente). En los casos de paro cardiorespiratorio, el tratamiento se inició antes de 45 minutos de acurrido el accidente en $2 \mathrm{y}$, en un tercer caso, 38 horas más tarde, por agravamiento del cuadro neurológico, con sospecha de hipertensión endocraneana. En los casos de sindrome de Reye y encefalitis se indicó el coma barbitúrico terapéutico por hipertensión endocraneana no manejable por medidas habituales. El diagnóstico de hipertensión endoctaneana fue clínico, sin medición instrumental.

La letalidad de este grupo fue de cuatro pacientes, y de los que sobreviven 3 quedaron en estado vegetativo. Sólo 2 fueron dados de alta en condiciones normales, sin secuelas neurológicas apreciables en el momento del alta.

Todos los pacientes, al iniciar el tratamiento de coma barbitúrico terapéutico, presentaron compromiso de conciencia importante: este fue definido como coma superficial cuando existía respuesta no atingente a estitnulos dolorosos y coma profundo si no habia respuesta a dichos estímulos. Los pacientes que sobrevivieron, tuvieron un tratamiento minjmo de 55 horas y se usaron dosis de thiopental que profundizaban el coma superficial o hacían desaparecer la respiración espontánea y las sincinecias, en caso de coma profundo. En general las dosis fueron moderadas, no superando $6 \mathrm{mg} x \mathrm{~kg} x$ hora. Al suspender el tratamiento en los pacientes que sobrevivieron sin secuelas, éstos demoraron 80 horas, en el caso de sindrome de Reye, y 63 horas, en ta asfixia por inmersión, en recuperar completamente la conciencia.

\section{DISCUSION}

En todos nuestros pacientes se uso thiopental sódico para producir coma barbitúrico terapéutico. Esta droga pertenece al grupo de thiobarbitúricos, en los cuales e] oxigeno del carbono 2 de ácido barbitúrico ha sido reemplazado por un sulfuro, dándole características de gran liposolu-

Tabla 2.

Coma barbitúcico terapéutico (thiopental) en sínd rome de hipertensión endocraneana o sínd rome anóx ico isquémico

\begin{tabular}{|c|c|c|c|c|c|c|c|c|c|}
\hline 1. Sexo & $\mathrm{F}$ & M & $\mathbf{M}$ & $M$ & $\mathrm{~F}$ & l: & $\mathrm{F}$ & $\mathbf{F}$ & $\mathrm{M}$ \\
\hline 2. Fdad & $2 \mathrm{a} 10 \mathrm{~m}$ & $1.48 \mathrm{~m}$ & $1 \mathrm{a} 7 \mathrm{~m}$ & $5 \mathrm{a} 10 \mathrm{ml}$ & $7 a 7 \mathrm{~m}$ & $2 m$ & $\mathrm{Ta} B \mathrm{nr}$ & lat & $1339 \mathrm{~m}$ \\
\hline \multicolumn{10}{|l|}{ 3. Diaynóstico } \\
\hline $\begin{array}{l}\text { 4. T jempo evolución cuadro } \\
\text { base previo a trat. (horas? }\end{array}$ & $?$ & 5 & 0.5 & 0,5 & 0,7 & 38 & 72 & 120 & 168 \\
\hline 5. Condicion inicio trat. & $\checkmark \mathrm{P}$ & $C P$ & $\mathrm{CS}$ & $\mathrm{cs}$ & C.P & $\mathrm{CP}$ & $\mathrm{Cs}$ & $\mathrm{CS}$ & $\mathrm{CP}$ \\
\hline $\begin{array}{l}\text { 6. Tourjeión terapia pretìa } \\
\text { (horas), nanitol, } \\
\text { esteroides, híperven. }\end{array}$ & 0.5 & - & - & 3 & 1,7 & 36 & 8 & 72 & 60 \\
\hline $\begin{array}{l}\text { 7. Dosis inicial } \\
(\mathrm{mg} \times \mathrm{kg} \times \mathrm{h})\end{array}$ & 9 & 2,8 & 3 & 9 & 2,5 & 3 & 3 & 3 & 3 \\
\hline $\begin{array}{l}\text { 8. Duración coma barb. } \\
\text { terapeut. (horas) }\end{array}$ & 75 & 60,5 & 87 & 31 & 0,6 & 80 & 55 & 59 & 7 \\
\hline 9. Condición al cgreso & $\mathrm{F}$ & EV & $\mathrm{N}$ & $\mathrm{EV}$ & $F$ & EV & $\mathrm{N}$ & $\mathrm{F}$ & $F$ \\
\hline
\end{tabular}

\begin{tabular}{|c|c|c|c|}
\hline ASF & $=a_{k}$ ix ia por inmessión & $\mathrm{F}$ & $=$ fallecido \\
\hline$C P$ & $=$ coma profunco & $N$ & $=$ normal \\
\hline $\mathrm{CS}$ & $=$ coma superficial & Paro $\mathrm{CR}$ & $=$ pare card iorespiratorio \\
\hline $\mathrm{F}, \mathrm{V}$ & $=$ estado vegetativo & & \\
\hline
\end{tabular}


bilidad, corta acción y breve latencia para empezar a actuar 18 .

La acción anticonvulsiva del thiopental sódico ya fue analizada en un trabajo anterior ${ }^{13}$, sin embargo debe recordarse sus posibles efectos colaterales por vía endovenosa y en altas dosis: bruscas bajas de presión arterial a veces severas, apnea y laringoespasmo, que hacen aconsejable su uso por expertos (en lo posible médico anes. tesista u otro médico habituado a manejar situaciones mencionadas), en unidades de tratamiento intensivo y con ventilación mecánica.

Se define el estado convulsivo como una convulsión generalizada o hemigeneralizada de duración mayor a 30 minutos, o varias crisis recurrentes, sin períodos intermedios de recuperación de la conciencia, cuya duración exceda 30 minutos. Tanto estudios clínicos como experimentales, han demostrado que este tipo de estado epiléptico debe ser controlado lo más pronto posible: si se permite que se extienda más de $60 \mathrm{~min}$, existe alto riesgo de daño neurológico permanente o muerte, de ahí que sea considerado como una emergencia médica. Existen otras formas de estado epiléptico: al estado epiléptico no convulsivo (como sucede en el estado de "pequeño mal" o ausencia y en el estado de epilepsia parcial compleja) y la epilepsia parcial continua, con preservación de la conciencia. En estas situaciones el pronóstico, en cuanto a las secuelas neurológicas y muerte, es mucho mejor y por lo tanto las medidas terapéuticas pueden ser menos agresivas y permiten mayor espera. Estudios experimentales han demostrado que se produce daño celular permanente en el hipocampus, amigdalas, cerebelo, tálamo y capas cortica. les medias, después de $60 \mathrm{~min}$ de estada convulsivo ${ }^{19-22}$. Este daño persiste aún si los animales de experimentación son ventilados artificialmente y los efectos metabólicos colaterales son cortcgidos. Por esto se sospecha que la muerte celular. en esta situación, es secundaria a mayor demanda metabólica, debido a actividad neuronal anormal y acumulación de algunas sustancias como ácido araquidónico, prostaglandinas, calcio, leucotrienos, entre otras. Por otro lado la duración de un estado convulsivo más allá de 60 min también conduce a complicaciones me tabólicas generalizadas como: acidosis láctica, aumento de presión intracraneana, hipoglicemia, hipertermia, sudoración excesiva, hipertensión arterial seguida luego de hipotensión y eventual shock. La excesiva actividad muscular puede producir también miolisis, mioglobinuria y trastornos renales secundarios.

Los hechos ya expuestos, hacen necesario, cuando las medidas habituales del tratamiento del estado convulsivo no logran controlar la condición en 60 min o menos, aconsejar el coma barbitúrico terapéutico, con thiopental sódico (en la forma indicada en la sección material y métodos) para controlar la crisis convulsiva rápidamente, para luego continuar con el microgoteo de mantención hasta completar 24 horas sin convulsiones. Junto con el coma barbitúrico de" berán mantenerse las restantes medidas adecuadas, que incluyen los anticonvulsivantes habitua. les (fenitoína u otros), además del tratamiento para edenı cerebral, vigilancia de presión arterial, pulso, respiración mecánica y en lo posible electroencefalograma continuo. Todas estas medidas permitirán controlar efectos colaterales negativos del uso de barbitúricos en altas dosis y acortar el tiempo del coma, evitando recurrencias de las crisis.

Nuestra experiencia mostró que el control del estado epiléptico a través de anestesia barbitúrica es efectivo: rápido y seguro. La letalidad de nuestra serie clínica $(40 \%)$ es mayor que la publicada recientemente, con porcentajes entre 6,6 y $25 \% 23,24$, esto puede ser atribuido a que nuestros pacientes constituyeron un grupo seleccionado refractario a terapias habituales y con severas enfermedades de base que agravaron el pronóstico. También nuestra serie clínica mostcó alta morbilidad (secuelas neurológicas), especialmente en pacientes con infecciones graves del sistema nervioso central, que probablemente provocaron daños independientes del estado epiléptico.

El grupo de pacientes con hipertensión endocraneana o síndrome anóxico isquémico o ambos, comprende condiciones clínicas diversas y de diferentes causas. También se agregan en la literatura casos de traumatismo encéfalocraneano severos: con presión intracraneana elevada ${ }^{11,25}$

Los barbitúricos pueden reducir el impacto de un episodjo isquémico anóxico a trayés de varios mecanismos: el metabolismo cerebral se reduce aproximadamente en $50 \%$ con grandes dosis de barbitúnicos 26 . Además aumenta la resistencia cerebro vascular, teduciendo el volumen sanguineo intracraneal y la presión intracraneana ${ }^{27}$; por otro lado desvían el flujo sanguíneo, de áreas que presentan buena respuesta vascular a otras áreas afectadas con relativa isquemia. Tambien estas drogas son efectivos anticonvulsivantes, e inmovilizan al paciente, ayudando en la respiración mecánica y cuidados de enfermería. Por otro lado se ha postulado la capacidad de estos fármacos de captar radicales libres y asi prevenir la destrucción celula $\mathbf{2}^{28}$.

La medición continua de presión intracraneana fue introducida hace mäs de 25 años en Francia y Suecia ${ }^{29}$ y luego adoptada en el resto del munđo. Esto llevó al reconocimiento de que 
la hipertensión endocraneana severa es común en traumatismos de cráneo, sindrome de Reye, encefalopatías post-anóxicas y metabólicas, encefalitis virales $y$ hemorragias intracraneanas. $A$ medida que la vigilancia de la presión intracra. neana se hizo mâs frecuente, los clínicos encontraron gran número de pacientes en que las medidas habituales (hiperventilación, infusión endovenosa de agentes osmótjcamente activos, glucocorticoides y drenaje de liquido cefalora. quídeo a través de un catéter intraventricular) eran inefectivas. La presión intractaneana elevada produce daño cerebral directamente a través de isquemia cerebral y por deformaciones de estructuras cerebrales. El coma barbitúrico al reducir ef matabolismo cerebral y el flujo sanguíneo cercbral; también reduce la presión intracraneana, lo que es más evidente cuando la presión intracraneana está elevada o es inestable, como conse. cuencia de aumento del flujo sanguíneo cerebral. Diversos trabajos en casos de traumatismos en. cefalocraneanos $^{8,11}$ y síndrome de Reye ${ }^{10.12}$ mostraron buenos resultados del coma barbitúrico en pacientes en quienes no disminuía la presión intracraneana con las medidas clásicas. En nuestros pacientes el manejo se hizo sin mediciones de presión intracraneana, usândose sólo parámetros clínicos. El escaso número de pacientes y la variedad de etiologias hace dificil sacar conclusiones válidas, pero se obse rvó mejor sobrevida en los pacientes con asfixia por inmersión y paro cardiorespiratorio. Sin embargo todos los pacientes de este último grupo presentaron secuelas neurológicas moderadas o graves. Es nuestra impresión que el inducir cama barbitúrico, significa perder la totalidad de parámetros clínicos, que se requieren para evaluar la condjción neurológica de nuestros pacientes y su evolución en cuanto a hipertensión endocraneana. Por esto es imprescindible la vigilancia continua de la presión intracraneana, que permitiría tomar decisiones en puntos tan importantes como: la dosis de barbitúricos necesaria para mantenerla bajo $15 \mathrm{~mm} \mathrm{Hg}$ con una presión arterial media. entre 60 y $90 \mathrm{~mm} \mathrm{Hg}$; el momento de interrupción de la terapia. an otras palabras, cuando la presión intracranéana menor de $15 \mathrm{~mm} \mathrm{de} \mathrm{Hg}$ ha sido mantenida por lo menos 48 horas, o el aumento de la presión intracraneana es menor de 3 milímetros de mercurio, después de inyectar $1 \mathrm{ml}$ de solución salina intraventricular, ó 0,1 de la misma en el espacio subaracnoíneo y, finalmente para detectar elevaciones de presión intracraneana al retirar los barbitúricos. Las únicas indicaciones para iniciar tratamiento inmediato con esta terapia, sin vigilancia de la presión intracraneana, serian la asfixia por inmersión y el paro cardiorespiratorio.
Estas consideraciones nos Hevan a recomendar que esta forma de tratamiento se realice en lugares que cuenten con ventilación mecănica, vigilancia de presión intracraneana, médicos familiarizados en el manejo de este tipo de pacientes (unidades de tratamiento intensivo) $y$, en lo posible, elementos de apoyo como tomografía axial computada y registro de potenciales evoca$\operatorname{dos}^{30}$, que reemplacen los parámetros clínicos $y$ elecrroencefalográficos, que se pierden con altas dosis de barbitứricos. Sólo en estas condiciones debería plantearse el uso de esta terapia.

\section{RESUMEN}

El uso de barbitúricos en altas dosis se ha empleado con aparente buen éxito en el manejo de hipertensión endocraneana, encefalopatía enóxica-jsquémica y estado convulsivo. Se estudiaron los resultados de esta terapia, con thiopental, en 2 grupos de pacientes hospitalizados en el hospital Roberto del Río. El primer lugar corresponde a 10 pacientes en estado convulsivo resistente a medidas terapéuticas habituales, los que respondieron en forma efectiva. rápida y segura. El otro grupo de 9 pacientes presentaron hipertensión endocraneana o encefalopatía hipóxica-isquémica o ambas. de diferentes etiologias, obteniéndose los mejores resultados en casos con asfixia por inmersión y paros cardiorespiratorios. Se analizan los esquemas de tratamiento, los efectos colaterales, letalidad y las secuelas neurológicas. El manejo de pacientes en coma barbitúrico terapéutico debe hacerse en unidades de tratamiento intensivo, con ventila. ción mecánica, medición continua de presión intracraneana (en pacientes con hipertensión endocraneana) y en to posible con electroencefalograma y potenciales evocados.

\section{REFERENCIAS}

J. Goldstein, A., Wells, B.A., Keats, A.S.: Increased toletance to cerebral anoxia by Pentobarbital. Arch. Int. Pharmacodyn. Ther. 161: 138, 1966.

2. Hoff. J.T., Smith, A.L., Hankinson, H.L. et al: Barbiturate protection from cerebral infarction in primates. Stroke 6: 28, 1975

3. Moseley, J.I., Laurent, J.P., Molinari, G.F.: Barbiturate attenuation of the clinical course and pathologic lisions in a primate stroke model. Neurology (Minneap.) 25:870, 1975.

4. Brann, A.S., Montalvo, J.M.: Barbiturates and asphyxis. Pediatr. Clin. North. Am. 17: $851,1970$.

5. Shapiro, H.M., Wyte, S.R., Loeser, J.: Barbiturateaugmented hypothermia for reduction of persistent intracraneal hypertension. J. Neurosurg. 40: 90 , 1974.

6. Marshall, L.F. Smith, R.W., Shapiro, H.M.: The influence of diurnal rythms in patients with utracrancal hypertension: Implications of management. Neurosurgery 2: $100,1978$. 
7. Shepiro, H.M., Galindo, A., Wyte, S.R. et al : Rapid intraoperative reduction of intracraneal pressure with thiopentone. Bu. J. Anaesth. 45: 1057, 1973.

8. Rockoff. M.A., Marshall, L.F., Shapiro, H.M.: High-dose barbiturate therapy in humans: a clinical review of 60 patients. Ann. Neurol. 6: 194, 1979.

9. Miller, D.J.: Barbiturates and raised intracraneal pressure (editorial). Ann. Neurol. 6: 193, 1979.

10. Morshall. L.F. Shapivo, H.M. Rausher, A. et al. Pentobabital thcrapy for intracrancal hypertension in metabolic coma: Reye's Syndrome. Crit. Care Med. 6: 1, 1978.

11. Morshall, L.F., Smith, Ralw, Shapiro, H.M.: The outcome with aggresive treatment in severe head injuries: acute and chronic basbiturate administration in the management of head injury. Neurosurg. 50: 26,1979 .

12. Venes, J.L., Shaywitz, B.A.+ Spencer D D.: Management of severe cerebral edem in the metabolic encephalopathy of Reye Johnson Syndrome. J. Neurosung. 40: 903, 1978.

13. Cerda, M. y col: Tratamiento con Thiopental del Estado Epiléptico en Niños. Rev. Chil. Pediatr. 54: 249,1983 .

14. Brown, A.S., Horton, J.M.: Status Epilepticus treated by intraverious infusion of Thiopentone Sodjun. Br. Med. J. 1: 27, 1967.

15. Feneck, R.O.: A case of status epilepticus Anacsthesis 36: 691, 1981.

16. Dundee, J.W., Gray R.C.: Thiopentone in Status epilepticus (letter) Br. Med. J. 1: 362, 196?.

17. Partinem, $M_{+}$Kovanen, J., Nilsson, $E$ : Stidus epilepticus treated by barbiturate anaesthesia with continuous monitoring of cerebral function. Br. Med. J. 282: 520,1981 .

18. Goodman, L.S., Gilman, A.: The pharmacological basis of Therapestics, Chapter 9, p.98. Fourth Edition, 1970. The Macmillan Company.

19. Kreisman, N.R., Rosenthal, M., La Manna, J.C., Sick, T.J.: Cerebral oxygenation during recurrent scizurc. In Delgado Escueta A.V., Wastorliain, C.,
Treiman, D.M., Porter, R.J.: Eds. Status epilepticus: basic mechanisms of brain damage and treatment. Advances in Neurology, New York: Raven Press, 1982, Vol. 34.

20. Meldrum, B.S, Vigoroux, R.A., Brierly, J.B. Systemic factors and epileptic brain damage: prolonged seizures in paralized artificially ventilated baboons. Arch. Ncurol. 29: 82..1973.

21. Chapman, H., Meldrum, B.S., Siesjo, B.K.: Cerebral metabolic changes during prolonged epileptic selzures in rats. J. Neurochen. 28: 1025, 1977.

22. Chapman, A., Inguar, M. Siesgo, B.K.: Free falty acids in the brain in bicuculline. induced status epilepticus. Acta Physiol. Scand. 110; 335, 1980

23. Celesia, G.: Prognosis in Convulsive Status Epilepticus. Adv. Neurology 34: 55, 1983.

24. Aicardi, $J$, Chevrie, $J . J$.: Consequences of Status Fpilepticus in Infants and Children. Adv. Neurology 34: 115, 1983.

25. Miller. J.D., Becker, D.P., Ward, J.D., et al. Significance of intracraneal hypertension in severe hcad injury. J. Neurosurg 47: 503, 1977.

26. Michenfetder, J.D.: The interdependency of cerebral functional and metabolic effects following massive doses of Thiopental in the dog. Anesthesiology $41: 231,1974$

27. Pierce, E.C., Lombertsen, C.J., Deutsh; S. et al: Cerebral circulation and metabolism during thiopental anesthesis and hyperventilation in man. J. Clin Inves 41: 1664, 1962 .

28. Siesgo, B.X., Cartson, C., Hagerdal, M. et al: Brain metabolism in the critjcally ill. Crit. Cate Med. 4: 283,1976

29. Lundberg, N.: Continuous recording and control of ventricular fluid pressure in neurosurgical practice. Acta Psychiatr. Newrol. Scand. 36: suppl. 149: $1-193,1960$.

30. Lundar, $T$, Ganes, T., Lindegaard, K.F.: Induced barbiturate coma: Mcthods for evaluation of patients. Crit. Care Med. 11: 559, 1983. 